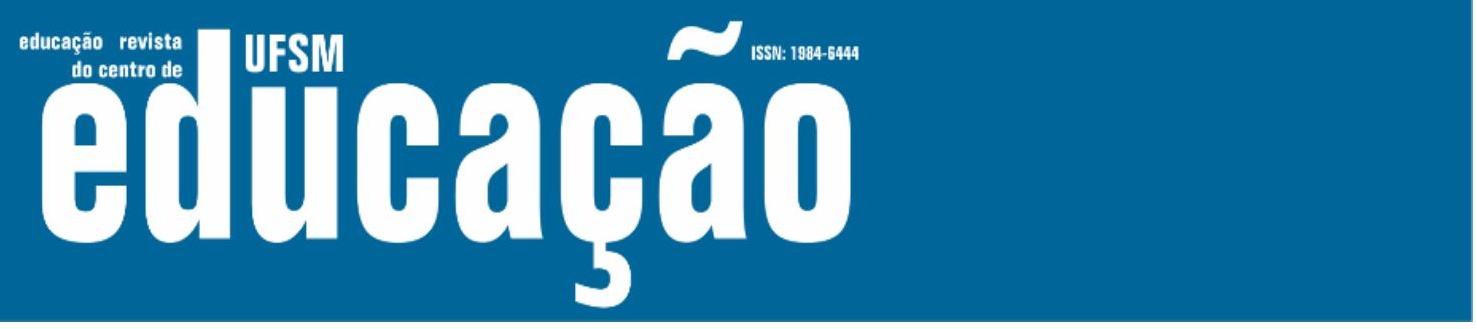

ISSN: 1984-6444 | http://dx.doi.org/10.5902/1984644467093

\title{
Riflessività e Ricerca Sociale: la produzione dialogica della realtà
}

\author{
Reflexivities and Social Research: the dialogic production of reality \\ Reflexividade e Pesquisa Social: a produção dialógica da realidade
}

Leandro Rogério Pinheiro

Universidade Federal do Rio Grande do Sul, Porto Alegre, Rio Grande do Sul, Brasil.

leandropinheiro75@gmail.com - https://orcid.org/0000-0001-5041-4939

\section{Enzo Colombo}

Universidade de Milão, Milão, Itália.

enzo.colombo@unimi.it - https://orcid.org/0000-0001-7231-5819

Recebido em 06 de agosto de 2021

Aprovado em 10 de agosto de 2021

Publicado em 04 de setembro de 2021

\section{RIASSUNTO}

Durante l'intervista abbiamo provato a problematizzare domande che riguardano la ricerca sociale su le infanzie e gioventù, evidenziando i processi riflessivi che costituiscono le azioni sociali contemporanee. In questo modo, invitiamo Enzo Colombo ad un'interlocuzione sulle diverse prospettive teorico metodologiche che portano alla luce il tema della 'riflessività', considerandola come categoria di interpretazione visto i processi di socializzazione e di individualizzazione ai giorni d'oggi. I contributi che risultano dalle risposte di Enzo Colombo ci portano dalle critiche epistemologiche e delineano didatticamente filoni interpretativi dell'azione riflessiva. Cioè: a) riflessioni dell'attore sociale su sé stesso e il contesto, in interazione con codici e attività; b) processo ricorsivo di incorporazione dei risultati dell'azione e della conoscenza; e c) pratica relazionale di produzione di sensi e azioni nell'interazione. In questo modo, inoltra i suoi argomenti alla discussione sulle condizioni socio-politiche per la produzione di una 'riflessività costruzionista', imprigionata ai contesti e ai dialoghi tra soggetti, attenta alla participazione della ricerca nella costruzione della realtà sociale.

Palavras-chave: Riflessività; Ricerca sociale; Realtà sociale.

\section{ABSTRACT}

During the interview, we aim to problematize issues pertinent to social research with/about childhood and youths, highlighting the reflexive processes that constitute contemporary social actions. Thus, we invite Enzo Colombo for a conversation about 


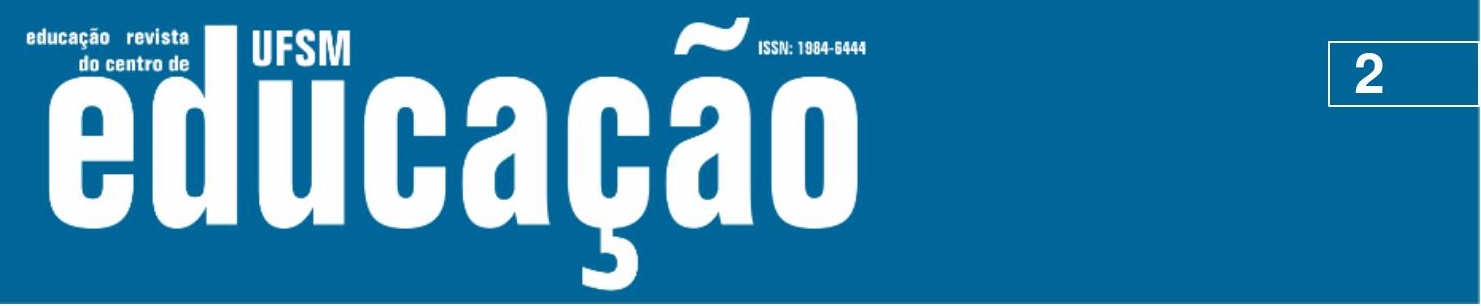

ISSN: 1984-6444 | http://dx.doi.org/10.5902/1984644467093

the different theoretical-methodological perspectives that bring up the theme of "reflexivity", considering it a category of interpretation in view of the current processes of socialization and individuation. The contributions raised by Enzo Colombo answers emerge from epistemological criticisms and didactically outline interpretative perspectives of the reflexive action, namely: a) the reflection of the social actor on $\mathrm{him} /$ herself and the context, interacting with codes and activities; b) the recursive process of incorporating results from action and knowledge; and c) the relational practice of producing senses and action in the interaction. Therefore, he continues his arguments to discuss the socio-political conditions to produce a 'construcionist reflexivity', held to the contexts and dialogues between the subjects, aware to the research participation in the construction of social reality.

Keywords: Reflexivities; Social research; Social reality.

\section{RESUMO}

$\mathrm{Na}$ entrevista, procuramos problematizar questões pertinentes à pesquisa social com/sobre as infâncias e juventudes, com destaque aos processos reflexivos que constituem as ações sociais contemporâneas. Dessa forma, convidamos Enzo Colombo para uma interlocução acerca de diferentes perspectivas teóricometodológicas que trazem à tona o tema da 'reflexividade', considerando-a como categoria de interpretação em vista dos processos de socialização e individuação na atualidade. As contribuições versadas nas respostas de Enzo Colombo trazem-no desde críticas de ordem epistemológica e delineiam didaticamente vertentes interpretativas da ação reflexiva, a saber: a) reflexões do ator social sobre si mesmo e o contexto, em interação com códigos e atividades; b) processo recursivo de incorporação dos resultados da ação e do conhecimento; e c) prática relacional de produção de sentidos e ações na interação. Assim, encaminha seus argumentos à discussão sobre as condições sócio políticas para a produção de uma 'reflexividade construcionista', entrelaçada aos contextos e aos diálogos entre sujeitos, atenta à participação da pesquisa na construção da realidade social.

Palavras-chave: Reflexividades; Pesquisa social; Realidade social.

\section{Introdução}

Enzo Colombo è professore e ricercatore del Dipartimento di Scienze Sociali e Politiche dell' Università degli Studi di Milano. Ha iniziato il suo lavoro in sociologia alla fine degli anni 1980, nel contesto educativo del "Laboratorio sul Mutamento Sociale (LAMS)", coordinato da Alberto Melucci. Come è stato affermato dallo stesso 


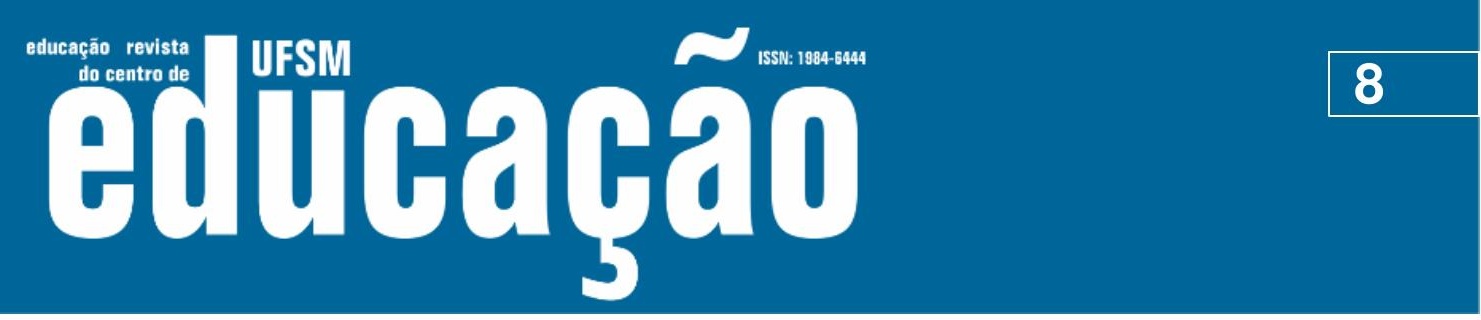

ISSN: 1984-6444 | http://dx.doi.org/10.5902/1984644467093

interessi particolari e che tende a strutturare la realtà secondo specifici rapporti di potere (ANDERSON, 2020). Sottolinea che la conoscenza è sempre coinvolgimento, partecipazione e responsabilità; che ogni conoscenza nel campo sociale che si vuole presentare come neutrale e universale non fa altro che occultare $i$ rapporti di potere che la rendono possibile e che contribuisce a riprodurre e consolidare. La riflessività situata invita a prendere posizione, a 'scendere in campo'. Denunciando la parzialità di ogni forma di conoscenza e la dipendenza di ciò che si può conoscere dalla posizione da cui si osserva, è un riflessività 'politica' che invita a un'identificazione esistenziale con i più marginali. La riflessività situata richiede molto più di una semplice elencazione delle caratteristiche identitarie del ricercatore; deve considerare modi alternativi di conoscenza, modi alternativi di azione, posizioni diverse - da quelle privilegiate di chi è investito istituzionalmente nel ruolo di 'ricercatore' - da cui guardare al campo di ricerca (SWEET, 2020). Deve assumere una posizione dal margine (bell hooks 1991), la posizione di chi è posto ai margini; perché il margine è una posizione di marginalità ma anche una posizione di distanza dalle logiche di potere e dal datoper-scontato su cui si regge lo status quo.

Bourdieu (2001) insiste sulla necessità di andare al di là di una riflessione sulle caratteristiche individuali del produttore di conoscenza per focalizzarsi sulle condizioni strutturali e sui rapporti di potere che definiscono la posizione del ricercatore nel campo della ricerca. Riflessività, in questo caso, implica una esplorazione sistemica delle «Categorie del pensiero impensate che delimitano il pensabile e predeterminano il pensato» (BOURDIEU, 1982, p. 10) mentre guidano la realizzazione pratica della ricerca sociale (WACQUANT, 1992, p. 33). Seguendo Bourdieu, la riflessività richiede non tanto una introspezione intellettuale quanto piuttosto un'analisi e un controllo sociologico permanenti della pratica di ricerca e di produzione di conoscenza. Riflessività implica, in questo caso, una riflessione sui condizionamento sociali di chi parla/fa ricerca/scrive, riconoscendo l'influenza che la sua posizione sociale ha su ciò che produce come conoscenza sociale. Implica riconoscere il fondamento sociale delle categorie che applichiamo alla realtà sociale per analizzarla; riconoscere che tali categorie hanno una genesi socio-storica situata, cioè sono inevitabilmente colorate 


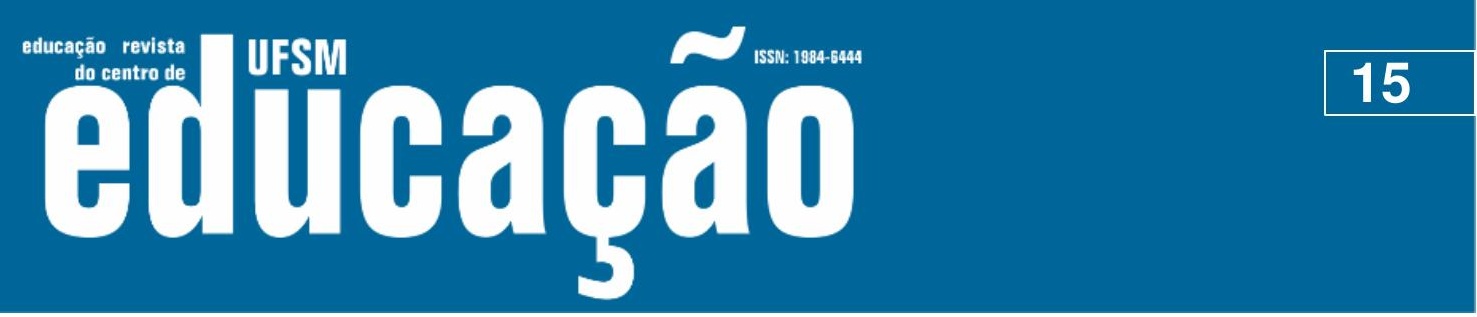

ISSN: 1984-6444 | http://dx.doi.org/10.5902/1984644467093

Reflexivity calls for the ethnographer to position herself. Recursivity positions her within processes she affects without controlling, within competing calls for response. Reflexivity asks what constitutes the ethnographer as a speaking subject. Recursivity asks what interrupts her and demands a reply. Thinking in terms of recursivity is a way to hold ethnography responsible for advocacy. Attention to recursivity foregrounds how every articulation - whether ethnographic or in direct advocacy - operates on previous articulations, inserting every move and every word within multiple discourses and worlds. (FORTUN, 2000, p. 13)

Questo non significa rinunciare a sviluppare l'auto-riflessività del ricercatore ma suggerisce di non limitarsi a ciò, perché il processo di ricerca sociale non si limita a cogliere una realtà che esisterebbe nella sua verità e nella sua autenticità al di sotto delle nebbie e degli inciampi che derivano dallo sguardo del ricercatore, inevitabilmente limitato dal suo posizionamento sociale. Piuttosto, il processo di ricerca è parte dei modi in cui, collettivamente e pubblicamente, ci confrontiamo e ci scontriamo nel tentativo di imporre un ordine - momentaneamente e parzialmente condiviso al fluire dell'esperienza.

Promuovere una riflessività relazionale significa riconoscere che il termine "riflessivo" si applica non al ricercatore ma alle relazioni tra ricercatore e altri soggetti partecipanti alla ricerca. Riflessività non si esaurisce completamente, dunque, né nella capacità di introspezione e di annullamento della distanza "esperienziale" tra osservatore e osservato - la capacità di non disgiungere mai il lavoro dalla condotta di vita, come auspica Wright Mills (1959) -, né nella capacità di esplicitare le condizioni e le scelte connesse al lavoro di ricerca. Si tratta piuttosto di favorire l'attivazione e il mantenimento di uno spazio dialogico, in cui gli assunti e la condizione situata della conoscenza possano essere evidenziate piuttosto che occultate e, quindi, essere sottoposte a revisione, confronto, confutazione.

L'idea di riflessività costruzionista vuole sottolineare come la produzione di sapere sociale necessiti di spazi istituzionali favorevoli al dialogo e al confronto. La conoscenza sociale nasce nel momento in cui azioni e interpretazioni si confrontano e il significato emerge nel momento della risposta che, per essere a sua volta compresa, attende un'ulteriore risposta. La conoscenza sociale si concretizza quando c'è una 
ISSN: 1984-6444 | http://dx.doi.org/10.5902/1984644467093

il nostro vocabolario condiviso per attribuire senso all'esperienza e contribuisce a configurare la realtà sociale in un modo specifico (un discorso che pure ha molteplici alternative, è situato e temporaneo, sottoposto a critica, un discorso che privilegia alcune voci e alcuni interessi, silenziandone altri, un discorso, infine, capace di creare forme specifiche di inclusione e di esclusione). Un'idea di riflessività ancorata alla prospettiva costruzionista tende a sottolineare che la conoscenza e la realtà del mondo non sono indipendenti dal coinvolgimento dei soggetti agenti e conoscenti, e che tale coinvolgimento dà forma alla realtà, all'azione e alla conoscenza. In questa prospettiva, riflessività indica il tentativo e la necessità di superare la distinzione tra realtà oggettiva e realtà soggettiva, tra osservatore e campo di ricerca. Come osserva Alberto Melucci (1998, p. 22), la svolta riflessiva

implica una ridefinizione profonda del rapporto tra l'osservatore e il campo. Si potrebbe dire che dalla dicotomia osservatore/campo si passa alla connessione osservatore-nel-campo. Tutto ciò che è osservato nella realtà sociale è osservato da qualcuno, che si trova a sua volta inserito in relazioni sociali e in rapporto al campo che osserva. Per queste ragioni il ruolo dell'osservatore e il rapporto tra l'osservatore e il cosiddetto oggetto di ricerca diventano un punto critico della riflessione sullo statuto della ricerca sociale. (MELUCCI, 1998, p. 22)

Penso, dunque, che sia interessante provare a sviluppare un'idea di riflessività che valorizzi la complessità e proceda secondo un metodo basato sulla ricerca della sintesi e del supplemento. Una riflessività che non si contrapponga secondo una logica di alternativa radicale alla riflessività essenziale dell'etnometodologia, alle forme tradizionali e moderne di riflessività, alla capacità di sottoporre ad analisi continua il modo in cui si sta osservando o intervenendo o alla capacità di introspezione dell'osservatore ma che sappia integrare queste prospettive includendo una riflessione sulle condizioni sociali che rendono possibile l'osservazione e sulle implicazioni che essa ha sulle sue stesse condizioni di possibilità e sulla realtà sociale che contribuisce a conoscere e a costruire.

Assumere la prospettiva di una riflessività costruzionista implica accettare un certo grado di relativismo; riconoscere che più che pervenire a conoscenze di leggi 


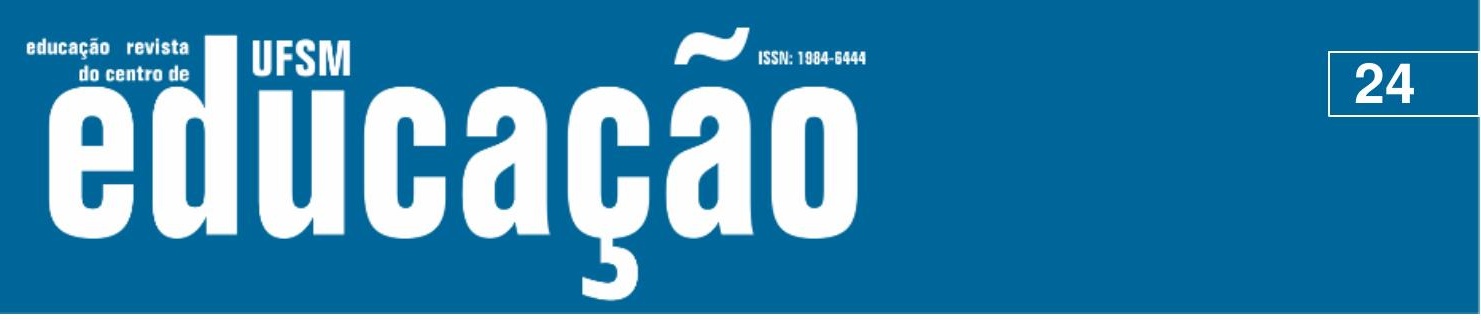

ISSN: 1984-6444 | http://dx.doi.org/10.5902/1984644467093

- un'affermazione, un'osservazione, una ricerca che sollecita attivamente la critica, il confronto, la discussione. É riflessiva - in senso costruzionista - una società che attiva spazi di discussione, che protegge la voce del dissidente, che assicura che ci possa sempre essere una risposta. La riflessività, come ricorsività, consiste principalmente in questo costante dialogo sociale sulla realtà sociale, in modo che si sia chiamati continuamente a dare ragione delle proprie ragioni. Richiede che siano presenti nello spazio pubblico prospettive e narrazioni diverse che consentano la presa di posizioni, l'assunzione di responsabilità, la messa in discussione della voce dominante.

Concretamente, cercare di produrre un sapere sociale riflessivo in prospettiva costruzionista significa mettere in atto mosse che favoriscano il dialogo (inteso anche come conflitto, disaccordo, confutazione), il confronto. Oltre a inglobare le indicazioni della riflessività metodologica (collocare il ricercatore nel campo, parlare delle modalità di produzione della ricerca), oltre 'prendere sul serio' gli attori sociali e considerarli produttori attivi di conoscenza, oltre a considerare le condizioni strutturali e le dinamiche contestuali che producono riflessivamente organizzazione sociale - tutti fattori a cui una buona ricerca sociale a fatica può rinunciare - un riflessività costruzionista cerca di mettere in atto degli accorgimenti che favoriscano la formazione di ambiti di dialogo e di confronto. Questo può avvenire sia nel campo di ricerca sia nel campo sociale. Nell'ambito specifico della ricerca e della metodologia delle scienze sociali, alcuni esempi possibili (ma è utile essere aperti e saperne inventare altri) possono essere azioni volte a favorire forme "istituzionalizzate" di riflessività relazionale, cioè forme "esterne" e "pubbliche" di confronto e di critica. Una prima possibilità potrebbe essere quella di istituire uno o più referenti esterni: colleghi che si fanno carico di seguire passo dopo passo l'evolversi della ricerca qualitativa. I referenti esterni potrebbero costituire utili attivatori di riflessività relazionale, sia stimolando il ricercatore a una maggiore consapevolezza rispetto alle proprie scelte, sia favorendo l'emergere di punti critici, sia, infine, assicurando una maggiore garanzia di controllo professionale sull'attività di ricerca. I referenti potrebbero inoltre trovare spazio autonomo nella restituzione dei risultati - nel testo finale della ricerca - ricostruendo, dalla loro prospettiva, la storia naturale della ricerca di campo. La scrittura della ricerca 


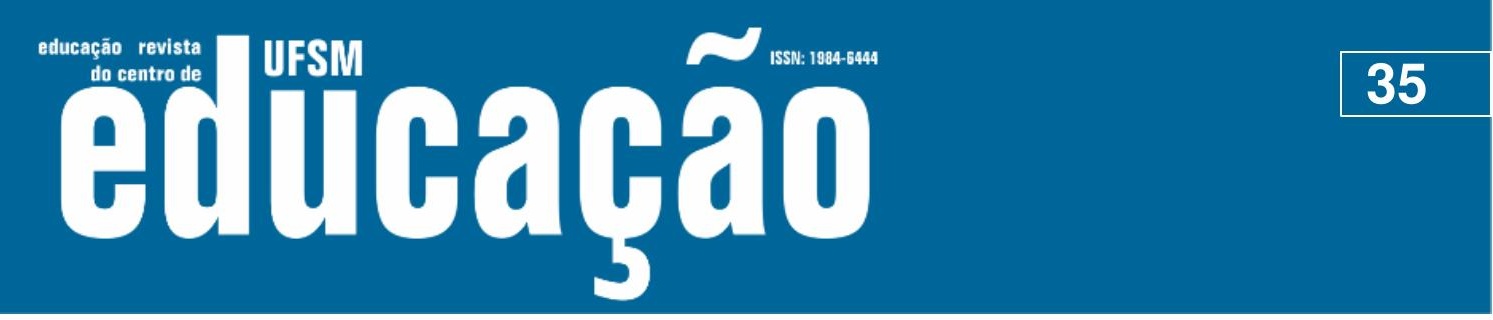

ISSN: 1984-6444 | http://dx.doi.org/10.5902/1984644467093

LYNCH, Michael. Against reflexivity as an academic virtue and source of privileged knowledge. Theory, Culture \& Society, 17 (3), p. 26-54, 2000.

MANNHEIM, Karl. The Problem of Generations. In: MANNHEIM, Karl. Essays on the Sociology of Knowledge. London: Routledge \& Kegan Paul Ltd, 1952.

MEAD, George Herbert. Mind, self and society. Chicago: The University of Chicago Press, 1934.

MELUCCI, Alberto. Verso una sociologia riflessiva. Bologna: II Mulino, 1998.

MELUCCI, Alberto. The playing self. Cambridge: Cambridge University Press, 1996.

MILL, John Stuart. System of logic. Cambridge University Press, 2012 [1843]. Disponível em: https://www.cambridge.org/core/books/system-of-logic-ratiocinativeand-inductive/290C43FBA4DC7022540D58E7EC49B1C2\#fndtn-contents

MORLEY, Christine. Critical reflexivity and social work practice. International Encyclopedia of the Social \& Behavioral Sciences, vol. 5, p. 281-286, 2015.

PAOLUCCI, Gabriella. Introduzione a Bourdieu. Roma-Bari: Laterza, 2011.

PELS, Dick. Reflexivity: One step up. Theory, Culture \& Society, 17 (3), p. 1-25, 2000.

POLLNER, Melvin. Left of ethnomethodology. American Sociological Review, 56, p. 370-380, 1991.

RAWLS, John. Justice as fairness. A Restatement. Cambridge MA: Belknap, 2001.

SALZMAN, Philip Carl. On reflexivity, American Anthropologist, 104 (3), p. 805-813, 2002.

SWEET, Paige L. Reflexivity in feminist standpoint: theory and Bourdieu. Gender \& Society, 34 (6), p. 922-950, 2020.

WACQUANT, Loïc. Introduction. In : BOURDIEU, Pierre; WACQUANT, Loïc. Réponse. Pour une anthropologie reflexive. Paris: Edition du Seuil, 1992, p. 08-37.

WEBER, Max. Die «Objektivität» sozialwissenschaftlicher und sozialpolitischer Erkenntnis. Archiv für Sozialwissenschaft und Sozialpolitik, 19, p 22-87, 1904 [traduzione italiana, 1958].

WEBER, Max. Wissenschaft als Beruf. Berlin: Duncker \& Humblot, 1919. 


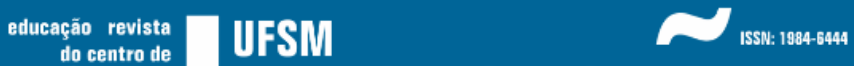

ISSN: 1984-6444 | http://dx.doi.org/10.5902/1984644467093

WOOLGAR, Steve. Knowledge and reflexivity: new frontiers in the Sociology of Knowledge. Sage: London, 1988.

WRIGHT MILLS, Charles. The sociological imagination. Oxford: Oxford University Press, 1959.

\section{(c) (7) (8)}

This work is licensed under a Creative Commons Attribution-NonCommercial 4.0 International (CC BY-NC 4.0) 